\title{
Réflexions sur le rapport néerlandais du NIOD : logique académique et culture du consensus
}

\section{Pieter Lagrou}

\section{(2) OpenEdition \\ 1 Journals}

Édition électronique

URL : http://journals.openedition.org/conflits/2205

DOI : 10.4000/conflits.2205

ISSN : $1777-5345$

Éditeur :

CCLS - Centre d'études sur les conflits lilberté et sécurité, L'Harmattan

Édition imprimée

Date de publication : 1 mai 2007

Pagination : 63-79

ISBN : 978-2-296-03084-8

ISSN : 1157-996X

Référence électronique

Pieter Lagrou, « Réflexions sur le rapport néerlandais du NIOD : logique académique et culture du consensus », Cultures \& Conflits [En ligne], 65 I printemps 2007, mis en ligne le 04 janvier 2010, consulté le 30 mars 2021. URL : http://journals.openedition.org/conflits/2205 ; DOl : https://doi.org/ $10.4000 /$ conflits.2205 


\section{Réflexions sur le rapport néerlandais du NIOD : logique académique et culture du consensus 1}

\section{Pieter LAGROU}

Pieter Lagrou enseigne l'histoire contemporaine à l'université libre de Bruxelles, il est chercheur à l'Institut d'histoire du temps présent à Paris. Parmi ses publications récentes : "La "Guerre honorable" et une certaine idée de l'Occident. Mémoires de guerre, racisme et réconciliation après 1945 ", in Marcot F., Musiedlak D. (dir.), Les Résistances, miroir des régimes d'oppression. Allemagne, France, Italie, Besançon, Presses universitaires de Franche-Comté, 2006, pp. 395-412 ; "L'bistoire du temps présent en Europe depuis 1945, ou comment se constitue et se développe un nouveau champ disciplinaire ", La Revue pour l'histoire du CNRS, $n^{\circ}$ 9, 2003, pp. 4-15.

T e 4 décembre 2006, lors d'une cérémonie officielle, le ministre néerlandais Le la Défense, Henk Kamp, a décerné des décorations militaires aux soldats et officiers qui avaient fait partie du Dutchbat, le bataillon néerlandais en charge de la protection de la population de l'enclave de Srebrenica. Le geste, qui a soulevé une vague de protestations, semble incompréhensible, vu l'échec dramatique du Dutchbat dans une mission dont l'issue fut le massacre de plus de 8000 personnes confiées à sa protection. Il semblerait que les autorités politiques néerlandaises ne se soient jamais posé la question de la responsabilité des militaires néerlandais dans le drame de Srebrenica, ou, si elles se la sont posée, qu'elles y aient répondu par un acquittement sans appel.

Pourtant, quelques mois seulement après la chute de l'enclave en juillet 1995, le gouvernement néerlandais a commandité un rapport d'enquête portant précisément sur les responsabilités néerlandaises dans ce drame. Ce rap-

1. Cette contribution est issue d'un article publié en néerlandais en 2003 : "Het Srebrenica-rapport en de geschiedenis van het heden ", Bijdragen en Mededelingen betreffende de Geschiedenis der Nederlanden, vol. 118, n 1-3, 2003, pp. 325-336. (Voir le résumé en anglais : http://www.knhg.nl/bmgn_art29.htm). Cet article a été entièrement transformé grâce à un échange critique soutenu avec Isabelle Delpla et la relecture attentive de Xavier Bougarel et Jean-Louis Fournel. Sans ces commentaires constructifs et l'aide, pour la traduction, de Mathieu Roeges, ce présent article n'aurait pas vu le jour. 
port, publié après plus de six ans d'enquête, est de loin, parmi tous les rapports commandés par des gouvernements ou parlements nationaux et organismes internationaux, le plus volumineux et par plusieurs aspects aussi le plus ambitieux. Eléphant blanc dans l'ensemble des contributions internationales visant à cerner le massacre de Srebrenica, le rapport défie toute catégorisation facile : rapport officiel, produit de recherche académique, effort de légitimation, enquête administrative, médiation politique, travail de mémoire. Tant par sa taille démesurée que par son statut ambivalent, ses conclusions ne se résument pas facilement et ne se prêtent pas non plus à une lecture critique univoque. Plus que par une analyse interne du texte, c'est par une contextualisation circonstancielle que cet article tentera de cerner ce rapport.

En effet, afin de comprendre l'organisation même du texte, la méthode de travail de l'équipe, le style de l'écriture et sa réception par la société néerlandaise, il faut l'insérer dans une tradition nationale très particulière de la gestion de débats de société par commission d'enquête interposée. L'enquête fut confiée au symbole même de cette tradition, l'Institut néerlandais de documentation de guerre (Nederlands Instituut voor Oorlogsdocumentatie, NIOD). Bien que l'ensemble des travaux précédents de l'institut portait sur des enquêtes de nature historique, par rapport à l'actualité brûlante dans laquelle le plongeait le rapport Srebrenica, ce texte ne peut être compris que dans la continuité de sa tradition et il porte toutes les traces d'un «style maison ». Ceci se rapporte à la méthode de travail et, en particulier, à l'organisation centralisée du travail collectif, ainsi qu'à une approche positiviste, qui se borne à la reconstruction des faits en évitant les débats d'interprétation. La prégnance des modèles d'interprétation hérités de l'historiographie du nazisme, premier domaine d'expertise du NIOD, semble pourtant secondaire par rapport à la dépendance inédite aux sources judiciaires et le contexte très particulier de la coïncidence chronologique d'une enquête de type historique avec un processus judiciaire en cours. Cela soulève bien entendu la question de la valeur ajoutée du travail du NIOD par rapport aux travaux du Tribunal international pénal pour la Yougoslavie (TPIY). Si le NIOD se donne une ambition pédagogique, il est ironique de constater que seules les versions néerlandaise et anglaise sont disponibles et que la version serbo-croate est toujours à l'état de projet, cinq ans après la publication du rapport ${ }^{2}$. Plus que de

2. Si le gouvernement néerlandais ne doit tirer qu'une conclusion de ce rapport, c'est qu'il est important que le travail de la commission soit accessible en Bosnie, afin de permettre que la recherche sur cette guerre civile soit poursuivie par des historiens locaux. L'expérience acquise et les sources accumulées doivent être partagées au moyen d'accords d'échanges et d'un soutien financier et logistique, afin qu'une recherche indépendante puisse se développer en Bosnie même. Puisque aucune communauté n'a la possibilité d'avoir un rapport critique à sa propre histoire tant que celle-ci est écrite à l'étranger, de surcroît à La Haye ou Amsterdam, il semble logique qu'une part des moyens considérables mis à la disposition de l'équipe du NIOD par le gouvernement néerlandais soit fournie à des équipes de chercheurs bosniens, avec les garanties scientifiques nécessaires. Il relève de la responsabilité du NIOD de veiller à ce qu'une telle suite soit donnée à leur recherche. Malheureusement, quatre ans après le fracas causé par la publication du rapport sur la scène politique néerlandaise, aucun projet ambitieux d'outreach 
pédagogie, serait-il donc question de légitimation ? A cet égard, on peut souligner le partage spécieux des responsabilités, focalisé sur les responsabilités en haut lieu, que ce soit à La Haye, New York ou Zagreb, tout en épargnant les militaires du Dutchbat eux-mêmes. On peut souligner le «timing » de la publication du rapport, qui a permis au gouvernement néerlandais de démissionner à trois semaines de la fin de la législature. On peut, in fine, souligner le caractère consensuel forcé de ses conclusions, qui semblent clairement vouloir clore le débat sur Srebrenica, plutôt que de fournir des éléments pour l'alimenter. Comme nous l'expliquerons en conclusion, cette culture du consensus et ce refus du débat contradictoire sont à la fois la caractéristique centrale de ce rapport et de la culture politique qui l'a produit.

\section{Un éléphant blanc?}

Parmi les différents rapports rédigés sur le massacre de Srebrenica de juillet 1995, celui présenté en avril 2002 par l'Institut néerlandais de documentation de guerre est exceptionnel à plusieurs titres ${ }^{3}$. Premièrement, par son impact immédiat : à la suite de la publication du rapport, le gouvernement néerlandais a assumé sa responsabilité collective pour les critiques formulées dans le rapport et a démissionné. Deuxièmement, par son ampleur : plus de 3000 pages en trois volumes et de nombreuses annexes, pour un total de presque 7000 pages ; une équipe qui, en

vers la société bosnienne n'a été concrétisé. Vu l'investissement considérable que représente la publication du rapport et de toutes ses annexes en néerlandais, ainsi que la mise en ligne de la version en anglais, il est incompréhensible que la version en serbo-croate, partiellement réalisée, ne soit toujours pas accessible, ni sous forme papier, ni sous forme électronique (communication téléphonique du responsable des relations publiques du NIOD à l'auteur, le 30 novembre 2006). Un tel choix semble particulièrement révélateur des priorités et des objectifs que le gouvernement néerlandais et le NIOD poursuivaient avec la publication du rapport.

3. Srebrenica, een "veilig" gebied. Reconstructie, achtergronden, gevolgen en analyses van de val van een Safe Area [Srebrenica, une "zone protégée ». Reconstruction, conséquences et analyses de la chute d'une Safe Area], NIOD, Amsterdam, Boom, 2002, 3 vol., 3392 p. Responsables: Hans Blom et Peter Romijn; chercheurs : N. Bajalica, G. Duizings, T. Frankfort, B. de Graaff, A. Kersten, P. Koedijk, D. Schoonoord, R. van Uwe, C. Wiebes ; soutien : M. van Kessel, E. Meents, $\mathrm{J}$. Vermolen; rédaction: P. Bootsma; annexes : Bootsma G., Het officiële NIOD-rapport samengevat [Le résumé du rapport officiel du NIOD], Leiden, Boom, 2002, 414 p. ; Naarden B., Beeld en Balkan. Waarneming en werkelijkheid van Zuidoost-Europa [Image et Balkans. Observation et réalité de l'Europe du Sud-Est], Amsterdam, Boom, 2002, 121p.; Schoonoord D., Dutchbat III en de bevolking : medische aangelegenheden. Bevoorrading door de lucht [Le Dutchbat III et la population : affaire médicales. Approvisionnement aérien], Amsterdam, Boom, 2002, 239 p.; Wiebes C., Intelligentie en de oorlog in Bosnië, 1992-1995. De rol van de inlichtingen- en veiligheidsdiensten [Renseignement et guerre en Bosnie, 1992-1995. Le rôle des services de renseignement et de sécurité], Amsterdam, Boom, 2002, 489 p. ; Duizings G., Geschiedenis en herinnering in Oost-Bosnië. De achtergrond van de val van Srebrenica [Histoire et mémoire en Bosnie orientale. Le contexte de la chute de Srebrenica], Amsterdam, Boom, 2002, 209 p. L'ensemble est accompagné d'un CD contenant huit fichiers word d'environ 1800 pages au total, dont les rapports de debriefing, des analyses supplémentaires des médias, une bibliographie commentée, des chronologies détaillées, une analyse de l'utilisation d'armes chimiques et une analyse du fonctionnement du ministère des Affaires étrangères. L'intégralité du rapport et sa table des matières détaillée sont accessibles sur le site du NIOD en néerlandais et en traduction anglaise à l'adresse suivante $:$ http://213.222.3.5/srebrenica/

4. http://www.knaw.nl/organisatie/pdf/Rapport_Evaluatiecommissie_NIOD_2003.pdf 
six années d'exercice, est passée de trois à douze chercheurs à temps plein ${ }^{4}$; un budget illimité qui, pour la dernière année seulement, avoisinait le million d'euros ${ }^{5}$. Troisièmement et finalement, par l'identité de l'institut qui a accepté de mener cette enquête commanditée par le gouvernement néerlandais : le NIOD, un institut créé en octobre 1945 pour étudier la période de l'occupation nazie aux Pays-Bas. En un demi-siècle d'existence, cet institut s'est exclusivement occupé d'enquêtes de nature historique sur la Seconde Guerre mondiale et, plus accessoirement, sur l'histoire coloniale et la décolonisation. Exhaustivité, impartialité, voire distance critique et historicisation du passé récent, voilà quelques-unes des attentes qu'un tel rapport mastodonte peut légitimement susciter, surtout par rapport à des commissions d'enquête parlementaires ou des audits internes d'organisations internationales, dont le mandat est beaucoup plus restreint dans le temps, les ressources plus modestes et le rapport avec le commanditaire institutionnel nettement plus étroit.

\section{Un pedigree}

Le rapport Srebrenica du NIOD s'inscrit dans un contexte national particulier dû, tout d'abord, à la légitimité incontestée de cet institut, qui, durant un demi-siècle, a pratiquement monopolisé la production historiographique (et la conservation des sources) sur la période de la Seconde Guerre mondiale. Les vingt-quatre volumes de L'Histoire du Royaume des Pays-Bas pendant la Deuxième Guerre mondiale [Het Koninkrijk der Nederlanden in de Tweede Wereldoorlog ${ }^{6}$ ], parus en livraisons annuelles entre 1975 et 1991 sous la seule plume du directeur de l'institut de l'époque, Louis de Jong, constituent un cas unique au monde par leur homogénéité monolithique et consensuelle, même si on les compare à des projets similaires menés par les académies des sciences des pays soviétiques. De Jong fut, par ailleurs, l'auteur de la première série documentaire télévisée retransmise en quelques dizaines d'épisodes pendant la première moitié des années 1960, et l'autorité morale incontestée dans tous les débats sur la guerre, la collaboration, l'épuration. Le «modèle de Jong » s'imposa par la suite comme une recette sûre pour la gestion consensuelle de débats de société, et donc pour leur dépolitisation. Une telle gestion par commission d'enquête interposée fut aussi critiquée comme une "mise en congélation » de ces débats. L'enquête sur les crimes commis lors de la répression du soulèvement en Indonésie en 1947-1949, commanditée en 1969, fut publiée en 1994, suffisamment tard pour que toute polémique soit éteinte et que les responsables politiques aient largement atteint l'âge de la retraite 7 . La question de la spoliation et de la restitution des biens juifs pendant l'occupation

5. http://www.mindef.nl/actueel/parlement/kamerbrieven/2001/4/181201_begroting.aspx

6. de Jong L., Het Koninkrijk de Nederlanden in de Tweede Wereldoorlog, 14 vol., La Haye, Martinus Nijhof, 1969-1991.

7. Voir van Doorn J., «Belast Verleden. Over de historisering van de publieke moraal », Nederlandse Organisatie voor Wetenschappelijk Onderzoek, La Haye, 2000. 
nazie fut confiée pendant les années 1990 à pas moins de six commissions d'enquête. Une septième se pencha sur les aspects non financiers du retour des déportés par l'entremise d'une fondation de recherche spécifique créée au sein du NIOD ${ }^{8}$. Au fur et à mesure que sa mission d'enquête fut élargie - des rapatriés juifs aux autres rapatriés d'Allemagne et ensuite des Indes - le projet recruta jusqu'à cinquante chercheurs.

Pourtant, la demande présentée en 1996 par le gouvernement néerlandais d'enquêter sur le massacre de Srebrenica était totalement inédite. Pour la première fois, le NIOD acceptait de produire un rapport d' « histoire du temps présent ", l'enquête démarrant seize mois seulement après l'événement 9 . A plusieurs reprises, il fut reproché à l'institut sa lenteur et la publication tardive des résultats de ses travaux. Pourtant, au regard de tous les antécédents, c'est la vitesse avec laquelle fut établi le rapport qui frappe, sept ans seulement après le déroulement des faits et six ans après le début de l'enquête. A titre d'exemple, le premier volume de De Jong parut trente ans après les faits décrits, le dernier volume parut quarante-six ans plus tard, et la commission sur les excès commis en Indonésie mit vingt-cinq ans à rendre publiques ses conclusions.

Lorsque l'institut accepta la «mission Srebrenica », les événements dans lesquels le massacre de la population de l'enclave s'inscrivait étaient encore en cours: bien que la guerre de Bosnie ait pris fin en décembre 1995, la Yougoslavie continuait de se déchirer, les Pays-Bas avaient toujours des troupes sur place et la plupart des responsables politiques et militaires néerlandais occupaient encore les mêmes fonctions. Alors que l'investigation battait son plein, certaines évolutions d'une nouveauté radicale se produisirent, comme l'éclatement d'un nouveau conflit au Kosovo en 1998, l'intervention de l'OTAN l'année suivante et les premiers jugements du TPIY à La Haye, jetant un nouvel éclairage sur les événements et introduisant de nouveaux paradigmes dans la manière d'envisager les interventions militaires et humanitaires. L'acceptation de cette tâche de recherche par un centre pourvu d'une longue tradition de respectabilité historique constituait une transgression des règles délimitant l'exercice de l'histoire contemporaine, imposant un délai de réserve équivalent au minimum aux délais d'accès aux archives publiques (trente ans pour le régime général, nettement plus pour des sources spécifiques telles que les archives judiciaires ou militaires). Même une institution équivalente au NIOD en France, l'IHTP (l'Institut de l'histoire du temps présent) qui travaille depuis les années 1960 en étroite collaboration avec le NIOD, et qui a inscrit depuis 1981 «l'histoire du temps présent » dans sa dénomination, ne

8. Voir Lagrou P., Compte rendu critique de Bossenbroek M., De Meelstreep. Terugkeer en Opvang na de Tweede Wereldoorlog, Amsterdam, Bert Bakker, 2001, 687 p. in Nieuwste Tijd. Kwartaalschrift voor eigentijdse geschiedenis, vol. 2, n 5 , décembre 2002, pp. 72-78.

9. L'auteur remercie Dick van Galen Last pour les dossiers de presse qu'il a eu la gentillesse de lui envoyer régulièrement. 
s'était jamais aventuré si près de l'actualité ${ }^{10}$. La témérité du NIOD, doyen des instituts historiques travaillant sur la Seconde Guerre mondiale en Europe, a été interprétée comme une imprudence folle, mettant en jeu sa légitimité scientifique, afin d'obtenir une nouvelle source de financement très importante et d'élargir son champ de compétences. Comment le NIOD pouvait-il croire pouvoir adopter la même distance critique et bénéficier de la même autorité consensuelle sur un conflit en cours, là où il avait mis un demisiècle à l'obtenir pour la période de l'occupation nazie ?

Partant de ces attentes, d'une part, et de ces appréhensions, de l'autre, comment peut-on évaluer cet exercice, unique en son genre, tant dans le contexte international que dans le contexte néerlandais?

\section{Un positivisme salutaire?}

Par rapport au caractère inédit du défi, le lecteur est de prime abord surpris par le style homogène et bien rodé tout au long des 3000 pages. Au niveau de la structure comme de l'argumentation, le document témoigne du "style maison » du NIOD et le canevas utilisé rappelle immanquablement celui de Louis de Jong dans L'Histoire du Royaume des Pays-Bas pendant la Deuxième Guerre Mondiale. Le sujet est découpé avec soin en chapitres et paragraphes, avec de nombreux renvois croisés. Chaque question bénéficie d'une introduction circonstanciée, consacrant une attention particulière à la présentation des sources, comme aux questions de chiffres, de dates et de chronologie. Le document ne s'égare ni dans les questions théoriques, ni dans les problèmes d'interprétation, et ignore l'apport d'autres contributions sur le sujet si elles dépassent le strict rapport aux sources. La manière très systématique dont le sujet a été décomposé témoigne d'une foi inébranlable en la force explicative de faits bien établis. La démarche de l'historien semble incontestable, tant qu'elle se borne à la reconstitution du «Wie es eigentlich gewesen ist » («Tel que cela a véritablement été ») de Leopold von Ranke.

Quelques questions importantes sont laissées ouvertes, et permettent d'émettre des hypothèses et d'envisager des scénarios alternatifs quant au développement de la situation à certains moments cruciaux. Il s'agit d'hypothèses sur le mode « what if », très proche du terrain : que serait-il arrivé si les blindés avaient été munis de canons plutôt que de mitrailleuses ? Si les membres du Dutchbat avaient tiré sur les Serbes depuis leurs positions de blocage ? Si une partie de la population de l'enclave n'avait tenté de rejoindre à pied les territoires sous contrôle bosniaque ? Ces hypothèses et alternatives sont ensuite globa-

10. Voir Lagrou P., «L'histoire du temps présent en Europe depuis 1945, ou comment se constitue et se développe un nouveau champ disciplinaire ", La Revne pour l'bistoire du CNRS, $n^{\circ}$ 9, 2003, pp. 4-15; Lagrou P. « De l'actualité de l'histoire du temps présent », Bulletin de l'Institut d'bistoire du temps présent, vol. 75, juin 2000, pp. 10-22. 
lement résumées au travers des différents niveaux de conclusion. Elles permettent de faire le point sur les faits révélés par les recherches et de démontrer comment ceux-ci suffisent à résoudre la plupart des questions restées en suspens. Du fait de ces longues marches d'approche, censées déblayer le terrain, d'une systématique d'acier et d'un souci de l'exhaustivité et du menu détail, la lecture des 3000 pages s'apparente finalement à une bataille d'usure. L'accumulation des données, tel un rouleau compresseur, finit par écraser le lecteur.

L'introduction place cette approche sous le signe du «fact finding » (" établissement des faits ») : il suffit d'établir sur le plan factuel ce qui s'est réellement passé pour arriver à une interprétation consensuelle. La vérité, une et indivisible, s'impose d'elle-même. La question délicate du rapport au commanditaire de l'étude - le gouvernement néerlandais - est balayée comme un faux problème entièrement réglé par le contrat établi au préalable, garantissant l'accès aux sources pour les chercheurs ainsi que leur indépendance scientifique. Il en va de même pour la question de la date de publication du rapport. Les six ans correspondent au temps réellement incompressible pour mener une telle enquête, point. Le fait que le rapport ait été publié deux semaines avant la fin de la législature, permettant ainsi au gouvernement de démissionner dans un grandiose geste de contrition publique parfaitement indolore, fut ensuite présenté dans les différentes déclarations de l'équipe et surtout de son porte-parole autorisé, Hans Blom, directeur du NIOD et de l'enquête, comme une pure coïncidence, objet d'une polémique totalement déplacée.

Or, la structure du rapport porte toutes les traces d'un processus de rédaction centralisé, à savoir que les auteurs des différents chapitres s'accordent, au terme de moult discussions, sur un texte commun qui ne supporte aucune divergence de vues, et se soumettent à une interprétation commune. Ceci suppose inévitablement un mécanisme d'arbitrage en cas de désaccord, hypothèse confirmée par la façon dont le directeur de l'institut assume l'ensemble des conclusions et par le fait que les chercheurs de l'équipe n'étaient pas autorisés à intervenir dans le débat public avant la publication finale du rapport.

La logique solide de ce canevas se révèle particulièrement efficace pour la reconstitution de certains événements. A titre d'exemple, la description de l'exode à pied vers Tuzla impressionne à la fois par la maitrise du ton de l'analyse dont le rapport fait preuve, par la multiplication des perspectives et des sources et par l'usage parcimonieux mais efficace de citations ${ }^{11}$. Cette partie n'est pas seulement un modèle de retenue dans la description d'événements insupportables, elle est aussi la démonstration convaincante qu'une reconstitution précise des événements peut apporter des réponses aux questions centrales émises par le rapport, sur le caractère prévisible ou non de certaines évo- 
lutions, sur les niveaux et les processus de prise de décision, sur la circulation des informations et les responsabilités qui en découlent.

\section{Modèles historiographiques ?}

A cet égard, il semble que l'équipe partage de façon implicite la confiance restaurée en un positivisme historique qui caractérise l'historiographie récente du génocide perpétré par les nazis. Les débats théoriques sur les questions de culpabilité, d'intention ou de fonctionnalité, sont pour la plupart stériles s'ils ne sont pas immédiatement confrontés à la réalité du terrain de la micro-histoire. Par sa chronologie méticuleuse et par l'analyse qu'elle fournit du niveau de commandement et de décision, la reconstitution des événements de Srebrenica par le rapport du NIOD vise à montrer que, bien que d'autres exécutions massives d'hommes aient déjà eu lieu dans ce conflit, cette mise à mort systématique et perpétrée à une telle échelle, n'a pas été planifiée longtemps à l'avance et de façon centralisée. Selon l'interprétation du NIOD, ce fut sur place, dans un processus de radicalisation où se sont conjugués antécédents et circonstances locales particulières, que la situation a pris cette tournure inédite. Cette approche rejoint par certains aspects les analyses des meilleurs historiens contemporains du génocide perpétré par les nazis, comme Christian Gerlach, Dieter Pohl, Christopher Browning ou Florent Brayard, développées près d'un demi-siècle après les faits, et qui portent sur la prise de décision des massacres dans le génocide nazi, notamment dans l'activité des Einsatzgruppen sur le front de l'Est dans la seconde moitié de l'année 1941, jusqu'au début de l'extermination massive par gazage pendant l'été $1942{ }^{12}$. Par rapport aux schématisations plus anciennes, qui partaient d'une conception « naïve » de la préméditation avec un processus décisionnel strictement hiérarchique, ces analyses insistent sur les échanges entre le terrain et leur hiérarchie, sur l'expérimentation et la découverte de nouveaux horizons du possible dans le massacre de masse, ainsi que sur la radicalisation des échelles du temps, d'un millénaire, dont le Reich se voyait disposer pour réorganiser le continent européen, à un an seulement pour réaliser ce crime dans sa totalité.

La question de l'influence éventuelle de l'historiographie de cet « autre génocide » dans l'analyse du massacre de Srebrenica par l'équipe du NIOD mérite qu'on s'y attarde. Tout d'abord, la «tradition maison » du NIOD concerne principalement l'histoire de l'occupation allemande des Pays-Bas,

12. Brayard F., La "Solution finale de la question juive». La technique, le temps et les catégories de la décision, Paris, Fayard, 2004 ; Browning C., Ordinary Men. Reserve Police Battalion 101 and the Final Solution in Poland, New York, Harper, 1992 ; Gerlach C., Kalkulierte Morde. Die Deutsche Wirtschafts- und Vernichtungspolitik in Weissfussland, 1941 bis 1944, Hambourg, Hamburger Edition, 1999 ; Pohl D., Nationalsozialistische Judenverfolgung in Ostgalizien 1941-1944: Organisation und Durchführung eines staatlichen Massenverbrechens, Munich, Oldenbourg, 1996. 
centrée sur les questions de résistance et de collaboration et très peu sur l'extermination des juifs et les crimes du nazisme en soi. D'ailleurs, la publication du rapport en 2002 a coïncidé avec la création d'un Center for Holocaust and Genocide Studies au sein du NIOD, et en collaboration avec l'université d'Amsterdam. Les Holocaust Studies ne faisaient donc pas partie d'une tradition ancienne du NIOD. Parmi les membres de l'équipe, seuls les responsables en chef Hans Blom et Peter Romijn étaient des historiens de la Seconde Guerre mondiale, alors que les chercheurs qui ont mené les enquêtes de terrain provenaient de tout autres horizons : histoire militaire, histoire $\mathrm{du}$ renseignement, histoire diplomatique, anthropologie des Balkans. Les références aux travaux historiques et socio-psychologiques sur le comportement humain en situation extrême sont récurrentes, mais elles ne semblent pas fondamentales pour l'interprétation.

C’est plutôt sur le plan méthodologique que l'équipe du NIOD semble avoir tiré des enseignements d'un demi-siècle de Holocaust Studies, par sa combinaison de perpetrator history, essentiellement fondée sur les sources produites à la fois pour et par le TPIY, et de victim history, surtout constituée de témoignages (oraux) de survivants. Pour l'histoire du génocide nazi, la dichotomie entre une histoire victimaire compatissante, en grande partie basée sur des témoignages tardifs, et une histoire des bourreaux, en très grande partie redevable aux sources et aux interprétations judiciaires, est critiquée depuis une quinzaine d'années. La représentation «minoritaire » du nazisme, comme étant formé d'une clique de responsables criminels autour de Hitler a en partie été le résultat du Tribunal militaite international de Nuremberg, qui s'est borné à juger deux douzaines de haut dignitaires nazis, et des procès organisés ensuite par la République fédérale d'Allemagne qui, dans son refus de toute rétroactivité de la justice de Nuremberg, a requalifié les crimes nazis comme crimes de droit commun relevant du code pénal de 1870. La question du rapport entre les matrices interprétatives du rapport du NIOD et celles de l'historiographie du nazisme revient donc en grande partie à celle de son rapport respectif aux sources judiciaires.

\section{Une histoire tributaire de la justice?}

La manière dont les sources du TPIY sont exploitées par le rapport est inédite. La lutte de compétences entre juges et historiens, qui fit couler tant d'encre dans le cadre des procès organisés depuis les années 1960 pour juger les crimes contre l'humanité commis pendant la Seconde Guerre mondiale, semble ici transformée. Bien que les historiens du NIOD soulignent à quel point il était important pour eux de ne pas être confondus avec les enquêteurs du tribunal, il ne semble pas que la collaboration entre justice internationale et recherche historique ait été fondamentalement conflictuelle. Que les historiens aient pu consulter les dossiers judiciaires, non pas quelques décennies après leur élaboration mais le plus souvent avant même l'ouverture des procès en question, est une donnée radicalement nouvelle, avec un impact fondamental sur la définition de la dis- 
cipline historique basée sur l'idée de délai de réserve et de séparation chronologique. Une réflexion sur de telles conditions d'accès aux archives et l'anéantissement de la distance temporelle qu'elles impliquent semble cruciale. L'axiome selon lequel l'historien ne se pencherait que sur des faits « anciens » et sur un ensemble d'événements qu'il considère comme "achevés» est-il une conséquence des limites empiriques de son travail ou bien est-il une nécessité intellectuelle ? Autrement dit : si l'accès aux archives judiciaires et aux archives gouvernementales était soumis à une tout autre législation, dans laquelle le délai de prescription pourrait être levé dans certains cas particuliers et pour un ensemble spécifique de chercheurs, la réserve que la science historique s'est imposée jusque là serait-elle automatiquement abolie ? La réponse à cette question est bien sûr indissociable de l'identification de ces « cas particuliers » et des conditions auxquelles cet «ensemble spécifique de chercheurs » serait tenu, vis-à-vis de l'ensemble des confrères comme de l'opinion publique, une question que le rapport NIOD n'aborde pas et sur laquelle nous reviendrons dans notre conclusion.

Or, la suppression de la distance temporelle entre l'action de la justice et celle des historiens du NIOD n'a-t-elle pas renforcé encore plus leur dépendance envers les sources et les interprétations judiciaires, comparativement aux historiens du nazisme ? Et, en fin de compte, est-ce l'approche historienne qui a changé, ou l'action de la justice internationale ? L'approche du TPIY diffère radicalement de celle du Tribunal de Nuremberg par le poids accordé aux témoignages de victimes et par l'utilisation des méthodes de la police scientifique dans l'établissement des faits : exhumations des fosses communes, identification des victimes, croisement des éléments de preuve ${ }^{13}$. A cet égard, le saut qualitatif dans les analyses du rapport du NIOD est tout aussi redevable au TPIY que les défauts d'une historiographie du nazisme pouvaient être attribués à Nuremberg.

Au niveau du fact finding, il est donc extrêmement difficile de départager les apports ponctuels du NIOD de celui, massif, du TPIY. Quelle peut alors être la valeur ajoutée de la démarche historienne revendiquée par le NIOD, par exemple, par rapport à la démarche judiciaire ? Initialement, le TPIY fut l'objet de beaucoup de critiques et de méfiance. En effet, la justice internationale semblait servir de simple pagne à une communauté internationale faisant savoir qu'elle n'était pas disposée à intervenir pour arrêter le déroulement des tueries, mais qu'elle recourrait par la suite aux principes des droits de l'Homme avec d'autant plus de conviction en organisant tribunaux et procès. L'historien américain Peter Maguire a désigné, non sans ironie, cette démarche de "strategic legalism 14 ». Pourtant, plus le processus judiciaire avance, plus les apports de la justice internationale apparaissent comme cruciaux. Les

13. L'auteur remercie Isabelle Delpla de lui avoir suggéré cette remarque.

14. Maguire P., Law and War, an American History, Columbia, Columbia University Press, 2001. 
polémiques troubles sur le nombre de victimes, sur l'identité des bourreaux ou même sur la réalité de certains événements sont effectivement un élément important dans la construction de mythologies nationalistes. Il n'est pas dénué de sens d'avancer qu'en ce qui concerne les massacres perpétrés pendant la Seconde Guerre mondiale sur le territoire yougoslave, le manque d'historiographie critique et d'établissement des faits élémentaires a contribué à la détérioration des relations entre groupes de populations, et ce plus particulièrement à partir des années 1980. Le TPIY, au contraire, a mis en lumière quantité de faits irréfutables concernant tant le nombre des victimes que les structures de commandement, il a identifié des coupables et il en a condamné un certain nombre. Du reste, la présence d'un représentant des Serbes de Bosnie lors des commémorations et des inhumations de victimes à Potocari le 11 juillet 2003 aurait probablement été impossible sans les efforts de la communauté internationale pour démontrer de façon incontestable la réalité des tueries.

Pour un tribunal, la reconstitution des événements est subordonnée à l'établissement de la culpabilité. Pour la recherche historique, la démarche est en principe exactement inverse. S'il y a débat sur la question de savoir si le jugement contribue à la réconciliation, il semble incontestable que l'établissement critique du déroulement factuel d'événements contribue à apaiser les rapports entre les populations. Encore faudrait-il que les historiens se donnent cette ambition. Dans le cas du rapport du NIOD, ceci impliquerait non seulement de dépasser sa mission d'établir les responsabilités néerlandaises dans le massacre - un dépassement réalisé à un tel point qu'il revient très largement à relativiser certaines responsabilités néerlandaises, comme nous verrons par la suite - mais encore de se donner pour ambition de nouer le dialogue avec les populations concernées.

\section{Nouveaux paradigmes?}

La reconstitution détaillée et essentiellement descriptive qu'ambitionne le rapport, donne également un aperçu peu reluisant de l'intervention internationale. La description concrète des humiliations vécues quotidiennement par les membres de la FORPRONU et de la perte totale de respect que celles-ci ont entraîné aux yeux des forces armées locales est bien plus évocatrice qu'une indignation rhétorique. La même démarche permet de déconstruire efficacement le mythe d'un adversaire militaire redoutable, en présentant la tactique serbe comme une combinaison fragile d'artillerie lourde et de terreur locale semée par des bandes paramilitaires composées de criminels notoires.

L'accumulation des données quant à la façon absurde dont les militaires néerlandais furent envoyés sans protection dans une souricière, avec des instructions totalement contradictoires, avec des structures de commandement opaques et éclatées entre Tuzla, Zagreb, New York et La Haye, sans moyens suffisants pour assurer la protection des populations civiles dont ils avaient la 
charge, sans renseignement ni même photos aériennes, et en dépendant pour leur ravitaillement d'une armée serbe dont ils étaient de fait devenus les otages, résonne comme une accusation d'incapacité collective, d'aveuglement et de paralysie de la quasi-totalité des instances nationales et internationales impliquées. Une partie des grandes réponses se dissimule en effet dans les petits détails, et ce même si le lecteur doit maintenir cette conviction tout au long des trois mille pages que compte le rapport.

Pourtant, une reconstitution historique ne peut se limiter à la simple addition de faits passés au crible de la critique, mais doit s'élaborer en fonction d'une argumentation et des questions posées au départ de la recherche. Le positivisme peut certes être salutaire, mais il ne constitue pas en soi un agenda de recherche. Bien que l'enquête du NIOD infirme le préjugé selon lequel une histoire aussi récente manquerait de sources, le cadre d'interprétation reste un plus grand défi. Ainsi, une grande part du rapport est consacrée à l'analyse du débat néerlandais sur l'envoi de troupes, incluant une analyse des médias et une étude approfondie des débats politiques au Parlement et des prises de position publiques des différents partis politiques, au gouvernement ou dans l'opposition. La question semble légitime, car il s'agit du cadre d'analyse par lequel se décrypte la politique intérieure. Cependant, ces très nombreuses pages nous apportent très peu, si ce n'est une confirmation de ce qui apparaît comme un consensus national, où tous et toutes semblent, dans les grandes lignes, plaider la même cause.

Le rapport est plus innovant dans son analyse du fonctionnement de la politique européenne. Les effets pervers du mécanisme de rotation de la présidence sont démontrés de façon accablante. L'enjeu de préservation, voire de renforcement, du prestige national pendant une présidence entraîne un réflexe d' « union sacrée ", mettant temporairement hors-jeu le cours normal du fonctionnement démocratique. La joute diplomatique pendant laquelle les chefs de gouvernement européens tentent de marquer des points à l'échelon national, sans vision globale et à plus long terme, n'a pas donné de résultats convaincants dans le domaine de la politique agricole commune. Lors de la première réelle explosion de violence en Europe depuis 1945, elle mena à un échec dramatique et impardonnable. Le rapport convainc le lecteur que les modèles d'analyse de la décision politique, traditionnellement utilisés par les historiens, ne sont plus d'actualité. Clairement, dans la gestion de la crise bosnienne vue de la Haye, il ne s'agit ni d'un débat national partisan, opposant, par exemple, socialistes et chrétien-démocrates, ni d'un enjeu de relations internationales classique, où des notions d'intérêt géopolitique, militaire ou économique seraient primordiales. La politique militaro-humanitaire apparaît comme une stratégie très indirecte dont les vrais objectifs sont un positionnement dans le concert européen et une gestion affective de l'opinion publique nationale. L'image dressée de l'actuel processus décisionnel européen est inquiétante par l'incapacité structurelle et le déficit démocratique qu'elle 
révèle. L'exemple du parcours pathétique d'un télégramme diplomatique métamorphoserait assurément le plus grand eurosceptique en partisan acharné d'une Constitution européenne cohérente et efficace. L'analyse fondée sur l'optique strictement nationale ne permettant plus de comprendre les processus dans lesquels le pays évolue, les historiens ont besoin de nouveaux paradigmes. Si le rapport du NIOD n'apporte pas de modèles d'interprétation cohérents face à cette nouvelle réalité de l'histoire, il a au moins le mérite de dresser un diagnostic.

La conclusion principale du volet international du rapport revient à un constat d'échec structurel dans la façon de gouverner l'Europe. Un échec amplifié par l'incompréhension transatlantique et par la paralysie chronique dont souffrent les Nations unies. Il semble donc que les leçons à tirer le plus urgemment du rapport se situent à ce niveau. Ceci peut surprendre lorsqu'on sait que l'enquête fut demandée par le gouvernement néerlandais et que les attentes du public se portaient spécifiquement sur les responsabilités néerlandaises. Le rapport souligne globalement qu'un petit pays, inséré dans un cadre européen dysfonctionnel, ne peut impunément pratiquer la géopolitique. Le gouvernement néerlandais ne disposait ni des renseignements suffisants pour se lancer dans cette aventure, ni des moyens militaires adéquats pour assumer les responsabilités qu'il avait lui-même sollicitées. De façon plus générale, il semble que les décideurs aient manqué de l'expérience nécessaire afin d'évaluer les risques de façon réaliste. Selon le rapport, le volontarisme néerlandais a aussi été le produit d'une certaine tradition d'autosuffisance, couplée à un esprit missionnaire, caractéristique de sa politique étrangère, mais recelant une réelle ignorance du contexte régional. La haute conception de l'innocence et de la supériorité morale de ce petit pays dans le concert des grands, avec sa tradition de tolérance et de neutralité, fut aussi une forme d'aveuglement sur ses capacités réelles à intervenir dans un conflit en dehors de ses frontières.

Une question que le rapport ne formule pas et qui semble pourtant incontournable est celle du manque d'expérience militaire qui ne permettait pas aux troupes néerlandaises d'assumer les responsabilités qu'elles ont acceptées. La description détaillée du comportement des militaires néerlandais lors des journées fatidiques de juillet 1995 soulève avec force la question de savoir si des militaires français ou britanniques, avec leur expérience et leur code de l'honneur, auraient réagi avec aussi peu de vaillance que ne le firent leurs collègues néerlandais. Les paragraphes consacrés aux troupes du Dutchbat sont sans doute les plus décevants du rapport. Les militaires néerlandais se trouvaient effectivement dans une position désespérée, douloureuse et humiliante, si bien qu'il semble facile a posteriori de les blâmer et de leur faire porter l'ensemble des responsabilités. Si le rapport ne minimise en rien l'action, ou plutôt l'inaction, des casques bleus néerlandais, il se garde systématiquement de formuler un certain nombre de conclusions sur leurs qualités morales et intellectuelles. Situant les responsabilités en haut lieu, dans les dysfonctionnements de la coo- 
pération gouvernementale et internationale, le rapport témoigne d'une certaine solidarité populiste avec «nos garçons " («onze jongens »), livrés à euxmêmes dans la jungle bosnienne, trahi par les hommes politiques qui les avaient, de façon irresponsable, envoyés dans cette souricière, traumatisés par leur propre impuissance.

La description de leurs contacts avec les belligérants, avec la population locale et entre eux, comme de leur attitude en situation de crise n'est pourtant pas glorieuse. Certes, un soldat n'est pas un héros professionnel. Pourtant, l'absence de toute ardeur résistante ou d'indignation combative est affligeante. Qu'un rapport officiel hésite à utiliser le terme de «lâcheté ", soit, mais l'évitement de toute critique explicite de leur comportement est à la limite de la compromission. Le très long développement sur les trois entrevues entre le commandant du Dutchbat, Thomas Karremans, et le général Ratko Mladic (pp. 2620-2643) et en particulier sur le fameux toast de Karremans (celui-ci n'aurait bu que de l'eau, comme si le contenu du verre amoindrissait la responsabilité du geste) pourrait être hilarant s'il n'avait pas comme arrière-fond le massacre de 8000 personnes. Les passages sur les incidents racistes au sein du Dutchbat sont d'une prudence très circonstanciée. Ceux sur la prostitution et le marché noir sont carrément évacués en note de bas de page ${ }^{15}$. Ils semblent pourtant avoir toute leur place dans une analyse de l'hostilité que les militaires néerlandais suscitaient parmi la population qu'ils auraient dû protéger, élément clef dans la compréhension des événements. A aucun moment les troupes néerlandaises n'auront été l'incarnation de ces valeurs que leur pays considère comme fondatrices de son identité, entraînant en conséquence la mort de ceux qui avaient placé en eux leur confiance. Cette responsabilité fut aussi individuelle, ce que le rapport n'admet pas.

\section{Le « modèle des polders » ?}

La description pour le moins clémente de l'attitude et du comportement des militaires du Dutchbat semble compréhensible, tant au niveau humain qu'au niveau politique. L'absence d'une distance temporelle et sociale, entre témoins et historiens, fut également préjudiciable lors d'autres entreprises de la Zeitgeschichte, qu'il s'agisse de la description euphémisante faite par Jean Norton-Cru des expériences d'anciens combattants de la Première Guerre mondiale, ou d'un certain type d'historiographie, parfois trop empreinte d'émotion, fondée sur les témoignages de survivants des camps de concentration. Il

15. Le rapport distingue entre «l'inconduite régulière (trafic de drogue, d'armes, prostitution, marché noir et contrebande) ", qui ne suscite aucun commentaire particulier, et l'inconduite motivée par une aversion particulière contre la population musulmane (p. 1550, voir aussi p. 1572). Le rapport est bien plus explicite sur «l'inconduite » des prédécesseurs canadiens du Dutchbat et suggère que les soldats bosniaques commandés par Nasser Oric ne devinrent les principaux clients de la prostitution dans l'enclave qu'après l'arrivée des troupes néerlandaises. 
semble presque inévitable que l'identification culturelle et émotionnelle ait été plus forte en interviewant les soldats néerlandais que ce qu'elle ne fut lors des entrevues en Bosnie et ailleurs avec leurs homologues et certains survivants, l'entremise de traducteurs introduisant encore un peu plus de distance. Cependant, il aurait fallu tenir compte de ce déséquilibre affectif lors de la rédaction du rapport, omission ou choix lourds de conséquences pour l'appréciation d'ensemble de ces 3000 pages.

Ceci nous ramène à notre question de départ quant à la nature du postulat d'une zone tampon entre observation et objet d'étude : celui-ci est-il uniquement inspiré par des circonstances pratiques qui ont rendu les sources inaccessibles jusqu'ici, et peut-il être réduit à néant dès lors que ces archives deviennent subitement accessibles, ou bien cette distance représente-t-elle une nécessité intellectuelle ? L'équipe du NIOD a bénéficié d'un effort exceptionnel de transparence de la part du gouvernement néerlandais. Si les Pays-Bas apparaissent en quelque façon comme modèle ou comme "pays pilote ", c'est assurément plus pour leur politique en matière d'archives que pour leur politique militaire. Cette transparence s'appliquait toutefois exclusivement aux membres de l'équipe, triés sur le volet et accrédités de l'équivalent néerlandais du « secret défense » français. Les documents étaient regroupés dans un étage spécialement sécurisé du somptueux bâtiment du NIOD, ancien siège rénové d'une banque coloniale donnant sur l'un des plus prestigieux canaux d'Amsterdam.

En conséquence, le rapport du NIOD n'est pas une contribution à un débat qui continue, ouvert à la contradiction, mais un terminus en soi, étape finale et verrouillée d'un processus autiste. Aucun historien, sociologue ou simple citoyen néerlandais, bosnien ou égyptien qui s'intéresserait à la tragédie de Srebrenica ne pourra obtenir l'accès aux mêmes sources pour de très longues années encore. Celles-ci sont d'ailleurs souvent indiquées de façon très cryptique comme «source confidentielle (note 14)» ou «entretien confidentiel (note 85)». Ceci prive le rapport d'un caractère proprement scientifique, puisqu'une analyse ne peut être considérée comme scientifique, dans la définition de Popper, qu'à la condition qu'elle puisse être vérifiée, contredite et potentiellement réfutée. Une lecture critique du rapport comme celle proposée dans cet article, forcément impressionniste, s'apparente à une forme très imparfaite de peer review. Plus fondamentalement, il verrouille de la même façon le débat démocratique.

Bien entendu, l'accès exclusif était une condition sine qua non à la recherche. L'esprit d'ouverture dont fit preuve le gouvernement néerlandais était déjà remarquable et l'on ne peut attendre d'aucun organisme qu'il ouvre au public sans plus de précautions ses archives les plus récentes sur un échec traumatisant. Une telle politique de transparence serait tout simplement inconcevable de la part du gouvernement français ou de l'ONU, par exemple. Pour l'équipe du NIOD, le choix revenait donc à accepter ces conditions ou ne pas mener la recherche et laisser reposer la question des responsabilités néerlan- 
daises dans le massacre de Srebrenica dans les boîtes et cartons d'archives, pour quelques décennies supplémentaires encore.

Accepter le mandat d'enquête demandait un courage certain, qu'il faut saluer. Malgré les critiques sur l'interprétation proposée par le NIOD, les apports du rapport dans la reconstruction des événements sont considérables. Pourtant, nous sommes d'avis que ce que nous avons désigné précédemment comme le «style maison » du NIOD n'a fait qu'amplifier les effets pervers de cette situation intrinsèquement problématique. Depuis que Louis De Jong s'est attelé à l'écriture de son Royaume des Pays-Bas pendant la Deuxième Guerre Mondiale il semble que le travail d'équipe du NIOD implique une sorte de centralisme démocratique, solidaire et unanime, "un pour tous, tous pour un », où l'individuel s'efface devant le collectif, et vice-versa. Du moment que l'on ne supporte aucune dissension dans la production historiographique, le travail d'équipe et le travail d'écriture individuelle sont constamment confondus. Dans le cas de De Jong, le travail collectif du NIOD disparaissait derrière sa signature personnelle, dans le cas du rapport Srebrenica, c'est les signatures individuelles qui disparaissent derrière un travail collectivement assumé.

Pourquoi un rapport de recherche devrait-il présenter des conclusions et des interprétations univoques, qui s'emboîtent à la manière de poupées russes, depuis les plus petits paragraphes jusqu'à l'ultime épilogue ? Pourquoi cette irrésistible propension au consensus, si bien que le moindre trouble doit être aplani au cours d'interminables réunions internes ? Le "style maison » du NIOD s’inscrit dans un modèle plus général, le «modèle des polders » («Poldermodel»), qui repose sur une culture du consensus négocié, que ce soit, historiquement, entre calvinistes, protestants réformés et catholiques, entre syndicats et patronat ou entre partis membres d'une coalition gouvernementale. Le modèle des polders est construit sur une société fortement segmentée à sa base, mais intégrée au niveau de ses élites. La culture du consensus implique aussi un malaise face au désaccord, une incapacité chronique à gérer les dissensions. Cette tradition contribue, par exemple, à ce qu'à notre connaissance, on ne trouve dans nul autre endroit qu'au Pays-Bas la pratique étrange qui consiste à soumettre avant sa publication un compte-rendu critique aux auteurs qui en sont l'objet ${ }^{16}$.

Dans la presse, il fut pourtant abondamment fait allusion à des conflits et des divergences d'interprétation au sein de l'équipe de recherche, qui auraient surtout porté sur la question des responsabilités locales. De façon révélatrice, ces divergences étaient mises en rapport avec les avatars de la vie privée de certains membres de l'équipe - idylles avec un officier du Dutchbat, ou avec des ressortissants serbes - comme si la dissension était en quelque sorte un acci-

16. Lorenz C., "Het "Academisch Poldermodel" en de "Westforschung" in Nederland ", Tijdschrift voor Geschiedenis, vol.118, n², 2005, pp. 252-270. 
dent de parcours, une anomalie. Que des désaccords aient pu exister au sein de l'équipe est pourtant plutôt rassurant, attestant que celle-ci était constituée de chercheurs capables de raisonner de façon autonome et qu'au sein du groupe, les relations n'étaient pas placées sous une autorité qui aurait rendu ces différences d'approche inadmissibles. Alors pourquoi cette situation, naturelle dans une démocratie pluraliste et dans un exercice de critique scientifique, n'a-t-elle pu s'exprimer de quelque façon que ce soit dans le rapport ? Même le TPIY adjoint souvent ses publications et ses arrêts de minority opinions. Etant donné que le rapport était conçu pour faire l'objet d'un débat parlementaire, le Parlement néerlandais et l'opinion publique néerlandaise n'auraient-ils pas été mieux servis par un rapport contradictoire, dans lequel plusieurs auteurs exposeraient diverses analyses et considèreraient le lecteur comme suffisamment adulte pour se forger ses propres opinions ? Mais surtout, le problème fondamental lié à l'impossibilité pour les personnes externes à l'équipe de contester les analyses en vérifiant les sources, n'aurait-il pas été réduit si l'on avait permis aux discussions internes de trouver un écho dans le rapport ? Ce penchant irrésistible pour le consensus apparaît d'autant plus ironique à la lecture même du rapport, où l'on insiste sur la façon consensuelle dont tous les segments de la vie politique néerlandaise ont plaidé, sans même y réfléchir, pour l'envoi de troupes, sans laisser d'espace au débat ou aux objections, qui auraient pu protéger le gouvernement contre son propre volontarisme et sa propre naïveté. Le remède à cet échec collectif et consensuel vient-il donc d'un mea culpa tout aussi collectif et consensuel, un verdict homogène prononcé par une équipe de chercheurs dont le Premier ministre tire ses conclusions sans autre forme de discussion? Le rapport n'aura donc pas servi à étayer le débat d'informations critiques, mais à le clôturer. C'est donc bien aux attentes du gouvernement néerlandais que le NIOD a effectivement répondu, en lui permettant, par un rapport et une démission, de tourner une fois pour toutes la page noire de Srebrenica. 